\title{
Transient reduction of visual distraction following electrical stimulation of the prefrontal cortex
}

\author{
Joshua D. Cosman, Priyanka V. Atreya, \& Geoffrey F. Woodman
}

Department of Psychology, Center for Integrative and Cognitive Neuroscience, Vanderbilt Vision Research Center, Vanderbilt University, Nashville, TN, USA.

Abbreviated title: ATTENTIONAL DISTRACTION AND TDCS

Address correspondence to:

Josh Cosman

PMB 407817

2301 Vanderbilt Place

Vanderbilt University, Nashville, TN 37240-7817

E-mail: joshua.d.cosman@vanderbilt.edu

Word Count: 1,918

Figures: 4

References: 19

Keywords: attentional capture | prefrontal cortex | attentional control | transcranial direct-current stimulation (tDCS) 


\begin{abstract}
The ability to overcome distraction is critical to a number of goal-directed behaviors, but information that is not relevant to our goals often captures our attention and distracts us from the task at hand. Neuroimaging work has demonstrated that activity in specific regions of the lateral prefrontal cortex (PFC) is related to the suppression of distracting information, implicating PFC as a critical node in the goaldirected control network. In the current work we asked whether applying transcranial direct-current stimulation (tDCS) to PFC would influence the likelihood of attentional capture by salient, task-irrelevant visual information encountered during visual search. Our results showed that anodal stimulation, relative to sham or cathodal stimulation, led to a transient decrease in attentional capture lasting approximately 15 minutes after stimulation. This provides causal evidence that PFC is involved in goal-directed control over distraction, and provides a basis for using PFC stimulation as a causal tool to understand deficits in goal-directed control in both neurologically healthy and impaired populations.
\end{abstract}




\section{Transient reduction of visual distraction following electrical stimulation of the prefrontal cortex}

Overcoming distraction by salient information is key to effective goal-directed attentional control, and distractibility characterizes a number of neurological disorders. As a result, determining which brain structures are necessary for overcoming distraction is important for understanding and remediating deficits when they occur. A number of studies have implicated specific regions of the parietal and prefrontal cortices in the ability to exert top-down control over distracting information across a number of modalities (see Gazzaley \& Nobre, 2012, for a review).

Of particular relevance to the current work, Leber (2010) demonstrated that the magnitude of pretrial activity in a specific region of prefrontal cortex, the middle frontal gyrus (MFG), predicted the likelihood that a salient distractor would capture attention during visual search, with increased pre-trial MFG activation leading to a decreased impact of a salient distractor on search performance (Leber, 2010). This suggests that the MFG is a critical node in the goal-directed attentional control network, serving to control access to limited capacity attentional mechanisms.

Here, we used a classic attentional capture task (Theeuwes, 1992) coupled with transcranial direct-current stimulation (tDCS) to examine whether it is possible to causally manipulate the magnitude of visual distraction by applying current centered over MFG. Briefly, low-amplitude application of tDCS leads to transient changes in the resting membrane potential of neurons under the site of stimulation, with anodal stimulation leading to a relative increase in resting membrane potentials and cathodal stimulation leading to a relative decrease (Bindman et al., 1964). Thus, if prefrontal cortex, and in particular MFG, is causally involved in goal-directed control over visual distraction, we would expect a transient decrease in attentional capture following 
anodal stimulation, and a transient increase in capture following cathodal stimulation, relative to sham stimulation.

\section{Method}

\section{Participants}

Eighteen volunteers with normal visual acuity and without color-blindness provided informed consent. Sample size was estimated on the basis of previous tDCS work in our lab showing effects of prefrontal stimulation and RT differences of a similar magnitude to those reported here (Reinhart \& Woodman, 2014).

\section{tDCS procedure}

A within-subjects design was employed in which each subject acted as their own control across conditions, receiving cathodal, anodal, or sham stimulation in different experimental sessions spaced 1-7 days (mean 3.2 days) apart, with stimulation type counterbalanced across days. The active electrodes (measuring $4.5 \mathrm{x}$ $4.5 \mathrm{~cm}$ ) were centered bilaterally at locations F3 and F4 of the standard 10-20 electrode system, a region corresponding to MFG (Herwig et al., 2003). Reference electrodes (measuring $11.0 \times 4.5 \mathrm{~cm}$ ) were placed on the contralateral cheeks.

Placement over MFG was verified by modeling current flow at locations F3 and F4 using established methods (Figure 2; Wagner et al., 2007; Sadleir et al., 2010). A realistic finite element model of the head was generated from the MNI T1-weighted MRI reference brain from the CURRY 6.0 multimodal neuroimaging software (Compumedics Neuroscan). Our forward computation using a finite element model was implemented in SCIRun (available as open source software: http://software.sci.utah.edu).

Participants were stimulated for 20 minutes at $1.0 \mathrm{~mA}$ in the anodal and cathodal conditions, and in the sham condition the stimulator was turned on at the 
beginning, middle, and end of the session for 30 seconds to simulate the feeling of the active conditions without delivering sustained current.
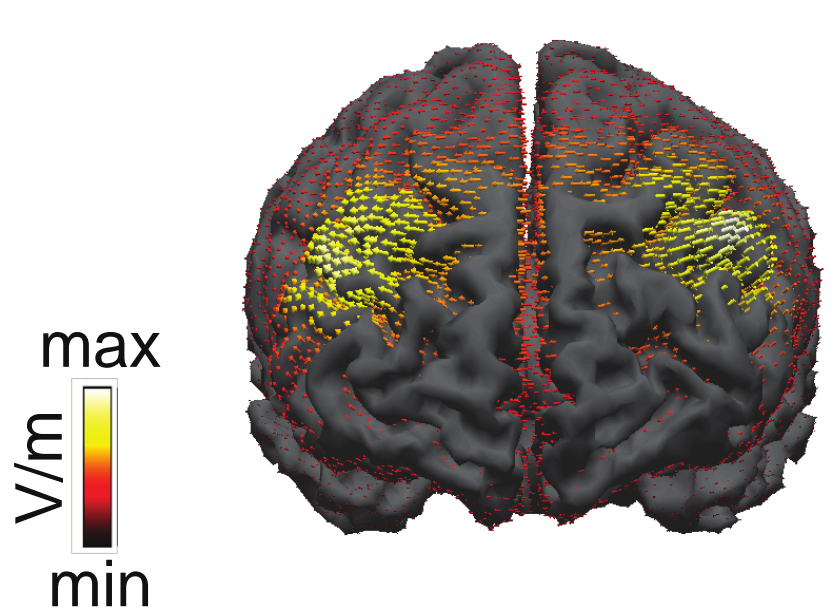

Figure 2. tDCS current flow diagram showing the distribution of current during active stimulation.

\section{Stimuli and Task}

Participants performed an adapted version of the additional singleton task (Theeuwes, 1992). Search arrays were viewed on a black background and consisted of twelve items placed in a circle centered on fixation (Figure 1); the items were equidistant from both each other $\left(1^{\circ}\right)$ and fixation $\left(6^{\circ}\right)$. Search items were either circles $\left(0.90^{\circ}\right.$ radius $)$ or squares $\left(1.6^{\circ} \times 1.6^{\circ}\right)$, and on each trial participants were asked to search for the item that differed in shape from the rest of the array, reporting the orientation of a line $\left(1.1^{\circ}\right)$ contained inside of it. On half of the trials, a color singleton distractor appeared at one of the non-target locations in the display (distractor present trials) and in the other half no color singleton appeared (distractor absent trials). All non-singleton items were drawn in light gray, and on distractor present trials the color singleton could randomly appear in red, green, blue, or yellow. 
Displays were always presented for $3000 \mathrm{~ms}$ followed by an intertrial interval that lasted $1000-1500 \mathrm{~ms}$, duration randomly jittered across trials, during which only the fixation point was visible. Participants were instructed to respond as quickly and accurately as possible. Participants were informed that the color singleton would never appear in the target location, and thus was a distractor that they should try to ignore. The task consisted of 3 blocks of 170 trials for a total of 510 trials.

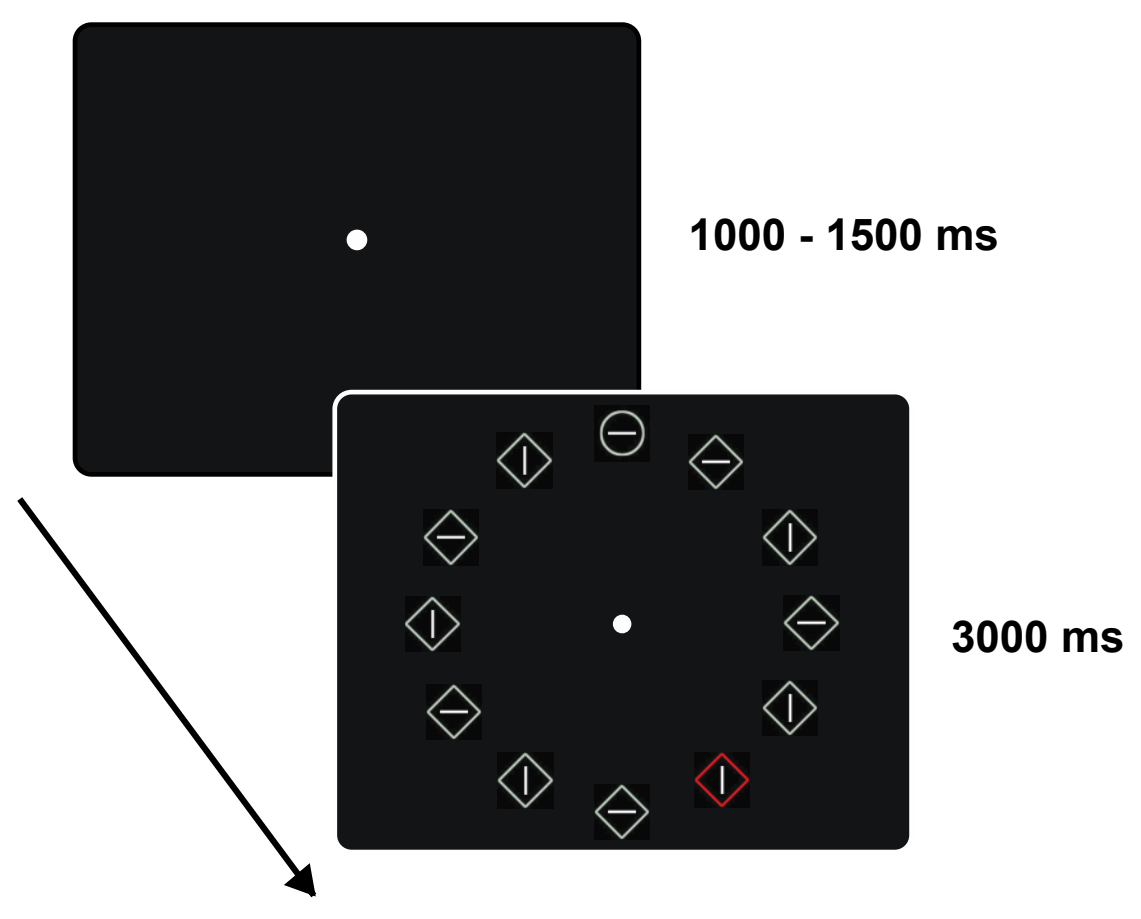

Figure 1. Stimulus displays showing a distractor present trial.

\section{Results}

Mean reaction times and error rates for each stimulation condition across blocks are shown in Figure 3. As can be seen in Figure 4, our tDCS manipulation of prefrontal activity decreased the ability of the singleton distractors to slow responses to the less salient targets in the anodal condition, with this effect being maximal in the first block of trials and decreasing thereafter. An omnibus three-way ANOVA, with stimulation condition (anodal, cathodal, or sham), block (first, second, or third), and 
distractor presence (present vs. absent) as factors revealed a significant main effect of block, $F(2,34)=41.3, p<.001, \quad \underset{p}{2}=.71$, and distractor presence, $F(2,34)=215, p$ $<.001, \quad \underset{p}{2}=.71$, and a significant three-way interaction, $F(4,68)=3.01, p=.02, \quad \begin{aligned} & 2 \\ & p\end{aligned}$ $=.15$, supporting the observation that the effects of stimulation and distractor presence depend on time following stimulation, as shown in previous work (Nitsche \& Paulus, 2001). No other main effects or interactions were significant ( $F s<.873$, ps $>$.49). An identical analysis performed on accuracy data revealed only a marginally significant main effect of distractor presence, $F(2,34)=4.21, p=.06, \quad{ }_{p}^{2}=.71$ (all other $F_{s}<1.32$, ps $>.27$ ).

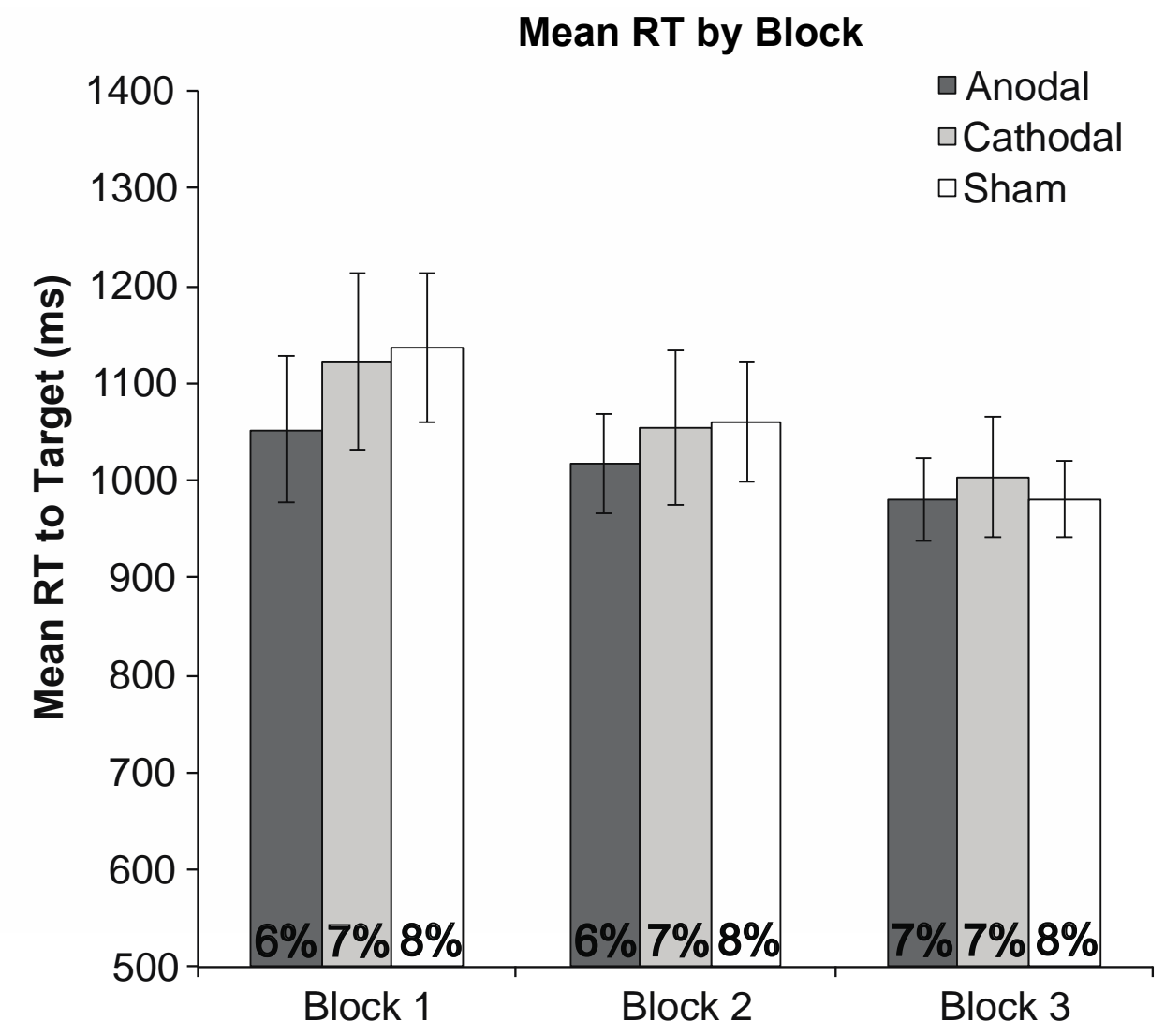

Figure 3. Overall reaction times and error rates (base of graph) for each stimulation condition, by block. Error bars indicate 95\% within-subjects confidence intervals (Morey, 2008). 
Given the time sensitivity of the observed tDCS effects, follow-up ANOVAs with stimulation condition and distractor presence as factors were performed on data from the first, second, or third block of trials individually in order to assess the time course of the tDCS effect. This analysis revealed a significant main effect of distractor presence across all blocks (first block, $F(1,17)=159, p<.001, \quad 2=.91$; second block, $F(1,17)=242, p<.001, \quad \underset{p}{2}=.94 ;$ and third block, $F(1,17)=61.6, p=.001, \quad \underset{p}{2}=.78)$. However, an interaction between stimulation condition and distractor presence was only observed during the first block of trials, $F(2,34)=3.38, p<.05, \quad{ }_{p}^{2}=.17$; (block two, $F(2,34)=.917, p=.41$, block three, $F(2,34)=.047, p=.95)$, suggesting that stimulation only influenced performance in the block of trials completed in the approximately 15 minutes following stimulation. There was no main effect of stimulation condition in any block (all $F \mathrm{~s}<1$ ). 


\section{Distractor Effect by Block}

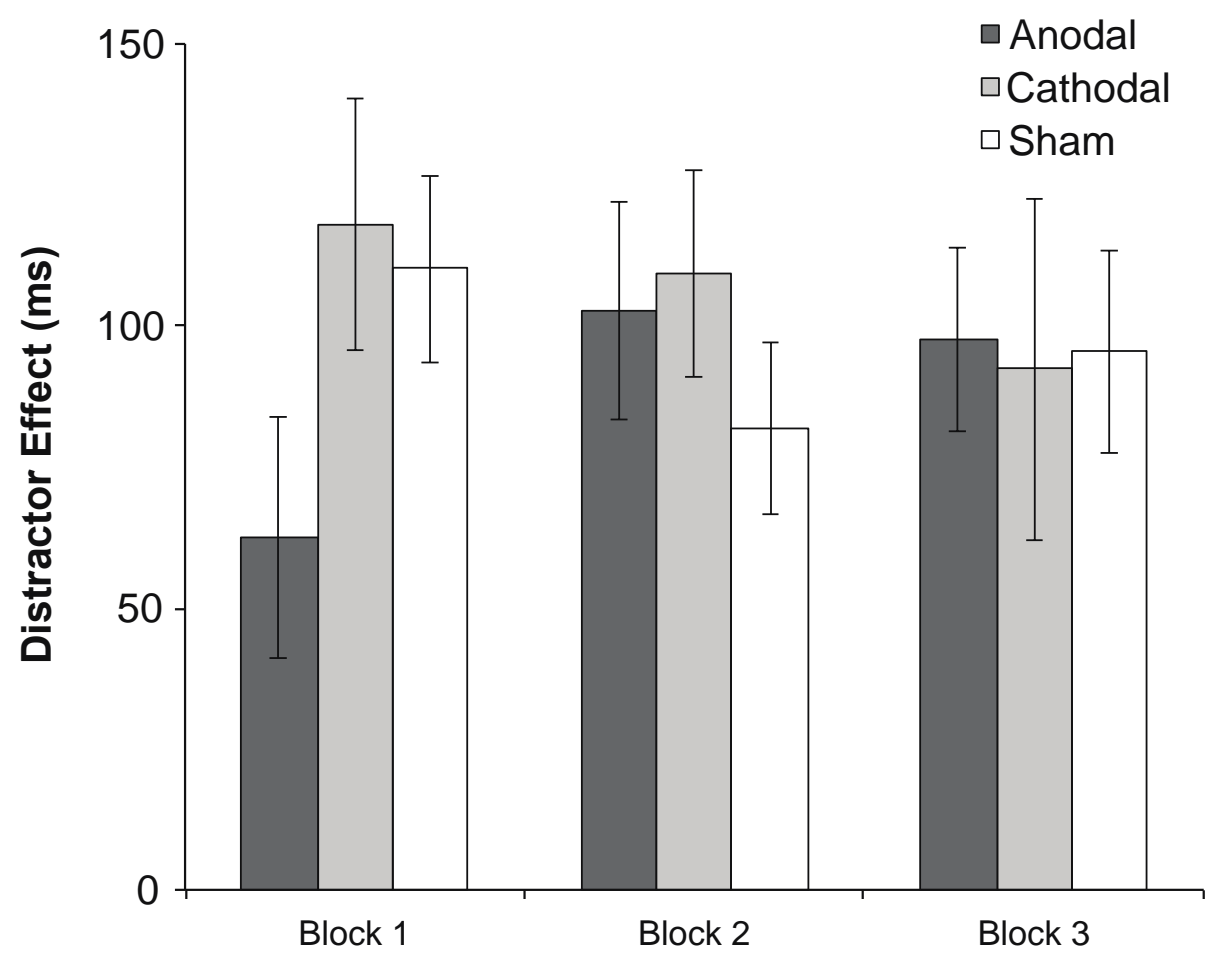

Figure 4. Magnitude of the distractor effect, calculated by taking distractor present minus distractor absent RTs, for each stimulation condition as a function of experimental block. Error bars indicate 95\% within-subjects confidence intervals (Morey, 2008).

Bonferroni-Holm corrected planned comparisons were performed on distractor effects (calculated by taking distractor present minus absent RTs; Figure 4) and revealed that this interaction was driven by a significant reduction in capture following anodal stimulation relative to both cathodal $(t=2.69, p=.03)$ and $\operatorname{sham}(t=2.47, p=.05)$ stimulation. Interestingly, distractor effects between cathodal and sham conditions did not differ $(t=0.69, p=.40)$, indicating that only anodal stimulation modulated attention in our task. This is in line with other recent work indicating an asymmetrical effect of anodal versus cathodal stimulation (see Nozari et al., 2014 for a review).

\section{Discussion}

Our results demonstrate that brief application of anodal tDCS centered over the middle frontal gyrus (MFG) transiently enhances the ability of individuals to 
overcome distraction by salient but task-irrelevant information, causally implicating the prefrontal cortex in the suppression of salient distractor. This complements previous TMS work causally implicating parietal cortex in this process (Hodsoll, Mevorach, \& Humphreys, 2009), and supports the notion that the parietal-prefrontal system is critical for the goal-directed control of attention.

Given that repeated stimulation can lead to longer-lasting residual effects of tDCS (see Monte-Silva et al., 2010), the current results suggest that repeated tDCS using the same montage employed here may provide a viable method for remediating distractibility due to neurological disorders or disease. Repeated tDCS has been shown to be effective for enhancing cognitive function in patients with depression (Fregni et al., 2006), and for recovery of motor function in patients with stroke (Boggio et al. 2007), and it has been suggested that a similar approach may be useful for remediating attentional symptoms of ADHD (Castellanos \& Proal, 2012).

Our finding that cathodal stimulation did not influence performance is of note. While it is unclear exactly why this asymmetry arose, the lack of an effect of cathodal stimulation is consistent with a number of previous studies (see Nozari et al., 2014). In the current work, we speculate that it is possible that there is an upper bound on the effect of a distractor stimulus on performance, such that the maximum distractor effect is reached even in the absence of stimulation. Alternatively, it may be that the physiological underpinnings of cathodal vs. anodal tDCS effects are asymmetrical with respect to the intensity or duration of stimulation required to generate a behavioral effect.

Finally, given Leber's (2010) demonstration that a salient distractor's ability to capture attention varies systematically with MFG activity, it seems likely that the results of the current experiment are due to a direct modulation of MFG activity via anodal tDCS. However, although current flow was centered on the MFG, it can be 
seen in Figure 2 that our tDCS montage led to significant current flow into other prefrontal regions important for both attentional control generally and distractor suppression specifically. In particular, the caudal end of the MFG includes the frontal eye field (FEF), a central node in the goal-directed attentional control network (Corbetta \& Shulman, 2002). Of primary relevance to the current work, neurons in the frontal eye field (FEF) of macaques show suppressed firing in response to a salient distractor item encountered in a visual search task nearly identical to that used here (Bichot, Rao, \& Schall, 2001), suggesting a possible role for FEF in the suppression of salient visual information. Likewise, functional imaging studies in humans have demonstrated increased activation in BA6, which is caudal and slightly inferior to the MFG, in the presence of salient distractors (deFockert et al., 2004, Lavie \& deFockert, 2006). Thus, our stimulation procedure may have led to a modulation of multiple attention-related prefrontal regions, and it is possible that each region may have been at least partially responsible for the effects observed here.

Regardless of the precise region(s) of prefrontal cortex modulated by our stimulation montage, this does not diminish the fact that anodal stimulation led to a transient reduction in visual distraction, and this work provides a basis for future studies combining tDCS with functional imaging to determine the network-level dynamics responsible for this effect. More generally, our results demonstrate that tDCS can be used as an effective tool for causally examining the mechanisms underlying visual distraction and attentional control. 


\section{Acknowledgements}

This work was supported by grants from the National Institutes of Health (F32-

EY023922 awarded to J.D.C ; R01-EY019882 and P30-EY08126 awarded to G.F.W.) the National Science Foundation (BCS-0957072 awarded to G.F.W.), and a Vanderbilt Discovery Grant. Thanks to Rob Reinhart for expert assistance with current flow analyses. 


\section{References}

Bichot, N.P., Rao, C., \& Schall, J.D. (2001). Continuous processing in macaque frontal cortex during visual search. Neuropsychologia, 39, 972-82.

Bindman, L.J., Lippold, O.C.J., \& Redfearn, J.W.T. (1964). The action of brief polarizing currents on the cerebral cortex of the rat during (1) current flow and (2) in the production of long-lasting aftereffects. Journal of Physiology, 172, 369382.

Boggio, P.S. et al. (2007). Repeated sessions of noninvasive brain DC stimulation is associated with motor function improvement in stroke patients. Restorative Neurology and Neuroscience, 25, 123-129.

Castellanos, F., \& Proal, E. (2012). Large scale brain systems in ADHD: beyond the prefrontal-striatal model. Trends in Cognitive Sciences, 16, 17-26.

Corbetta, M., \& Shulman, G.L. (2002). Control of goal-diredcted and stimulus-driven attention in the brain. Nature Reviews Neuroscience, 3 201-215.

De Fockert, J., Rees, G., Frith, C., \& Lavie, N. (2004). Neural correlates of attentional capture in visual search. Journal of Cognitive Neuroscience, 16, 751-9.

Fregni, F., Boggio, P.S., Nitsche, M.A., Rigonatti, S.P., Pascual-Leone, A. (2006). Cognitive effects of repeated sessions of transcranial direct-current stimulation in patients with depression. Depression and Anxiety, 23, 482-484.

Gazzaley, A., \& Nobre, A. C. (2012). Top-down modulation: bridging selective attention and working memory. Trends in Cognitive Sciences, 16, 129-135.

Herwig, U., Satrapi, P., \& Schönfeldt-Lecuona, C. (2003). Using the international 1020 EEG system for positioning of transcranial magnetic stimulation. Brain Topography, 16, 95-99.

Hodsoll, J., Mevorach, C., \& Humphreys, G. W. (2009). Driven to less distraction: rTMS of the right parietal cortex reduces attentional capture in visual search. Cerebral Cortex, 19(1), 106-114.

Lavie, N., \& De Fockert, J. (2006). Frontal control of attentional capture in visual search. Visual Cognition, 1, 863-876.

Leber, A.B. (2010). Neural predictors of within-subject fluctuations in attentional control. Journal of Neuroscience, 30, 11458-11465.

Monte-Silva K., Kuo M.F., Liebetanz D., Paulus W., Nitsche M.A. (2010). Shaping the optimal repetition interval for cathodal transcranial direct current stimulation (tDCS). Journal of Neurophysiology 103, 1735-1740

Morey, R.D. (2008). Confidence intervals from normalized data: A correction to 
Cousineau (2005). Tutorial in Quantitative Methods for Psychology, 4, 61-64.

Nitsche, M.A., \& Paulus, W. (2001). Sustained excitability elevations induced by transcranial DC motor cortex stimulation in humans. Neurology, 57, 1899-1901.

Nozari, N., Woodard, K., Thompson-Schill, S.L. (2014). Consequences of cathodal stimulation for Behavior: When does it help and when does it hurt performance. PLoS One, 9, e84338.

Reinhart, R.M.G., \& Woodman, G.F. (2014). Causal control of medial-frontal cortex governs electrophysiological and behavioral indices of performance monitoring and learning. Journal of Neuroscience, 34, 4214-4227.

Sadleir, R. J., Vannorsdall, T. D., Schretlen, D. J. \& Gordon, B. (2010). Transcranial direct current stimulation (tDCS) in a realistic head model. Neuroimage, $51,1310-1318$.

Theeuwes, J. (1992). Perceptual selectivity of color and form. Perception \& Psychophysics, 51, 599-606.

Wagner, T. et al. (2007). Transcranial direct current stimulation: a computer-based human model study. Neuroimage, 35, 1113-1124. 